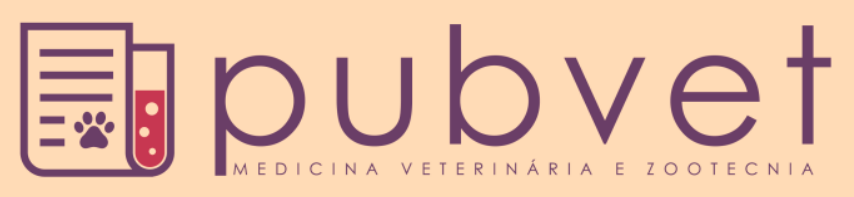

https://doi.org/10.31533/pubvet.v12n8a144.1-6

\title{
Tripanossomose bovina: revisão
}

\author{
Pedro Henrique Vieira Germano ${ }^{1 *}$, Alex André da Silva ${ }^{2}$, Gertrud Elisa Campos \\ Edler $^{3 \oplus}$, Marcelo Coelho Lopes ${ }^{4}$, Talita Cristina Modesto ${ }^{50}$, Junior Artur dos Reis ${ }^{6 \oplus}$ \\ ${ }^{I}$ Médico veterinário, Patos de Minas-MG, Brasil. \\ ${ }^{2}$ Discente do curso de medicina veterinária no centro universitário de Patos de Minas-UNIPAM, Patos de Minas-MG, Brasil. \\ ${ }^{3}$ Departamento de veterinária do centro clínico veterinário CCV-UNIPAM, Patos de Minas-MG, Brasil. \\ ${ }^{4}$ Médico veterinário residente em patologia animal, universidade de Uberaba-UNIUBE, Uberaba-MG, Brasil. \\ ${ }^{5}$ Médica veterinária residente em patologia animal, universidade federal de Uberlândia-UFU, Uberlândia-MG, Brasil. \\ ${ }^{6}$ Médico veterinário, Patos de Minas-MG, Brasil. \\ *Autor para correspondência, E-mail: pedro.vieiral1@yahoo.com.br
}

RESUMO. A tripanossomose bovina é uma doença de ocorrência global, o agente etiológico de maior relevância para bovinos é o Trypanosoma vivax. Foi registrada pela primeira vez no Brasil, em 1972, e recentemente, tem sido reportada em todo país através de surtos, acometendo tanto bovinos leiteiros quanto bovinos de corte, causando grandes prejuízos aos pecuaristas. Por se tratar de uma doença que por muito tempo vinha sendo negligenciada, essa enfermidade se tornou um grave problema. O objetivo do estudo é fazer uma revisão abordando os aspectos clínicos e patológicos bem como os fatores de risco, as técnicas de diagnóstico, tratamento e controle da doença gerando informações pertinentes para acadêmicos, profissionais e produtores rurais.

Palavras chave: controle, diagnóstico, sinais clínicos, Trypanosoma vivax

\section{Trypanosomiasis bovine: review}

ABSTRACT. The bovine trypanosomiasis is a worldwide occurring disease, the most relevant etiological agent for bovines is the Trypanosoma vivax. Its first record in Brazil was in 1972 and recently has been reported all over the country through outbreaks. Affecting both dairy and beef cattle, causing major losses to the cattle ranchers. Because it's a disease that, for a long time, was neglected, this disease had become a huge problem. The objective of the study is to make a review addressing the clinical and pathological aspects, as well as the risk factors, the diagnosing techniques, treatment and control of the disease generating relevant information for academics, professionals and farmers.

Keywords: control, diagnosis, clinical evidences, Trypanosoma vivax

\section{Tripanosomiasis bovina: revisión}

RESUMEN. La tripanosomiasis bovina es una enfermedad de aparición global, el agente etiológico de mayor importancia para los bovinos es el Trypanosoma vivax. Se registró por primera vez en Brasil, en 1972, y recientemente, ha sido reportada en todo el país a través de brotes, afectando tanto bovinos lecheros como bovinos de corte causando grandes pérdidas a los ganaderos. Por tratarse de una enfermedad que por mucho tiempo había sido descuidada, esta enfermedad se convirtió en un grave problema. El objetivo del estudio es hacer una revisión abordando los aspectos clínicos y patológicos así como los factores de riesgo, las técnicas de diagnóstico, tratamiento y control de la enfermedad generando informaciones pertinentes para académicos, profesionales y productores rurales.

Palabras clave: control, diagnóstico, signos clínicos, Trypanosoma vivax 


\section{Introdução}

Tripanossomose bovina é uma doença causada por um grupo de protozoários patogênicos do gênero Trypanosoma, que têm larga distribuição e importância econômica mundial (Adam et al., 2012; Batista et al., 2008).

Trypanosoma vivax (T.vivax) é um parasita originário da África, onde acarreta grandes restrições à produção de ruminantes, No continente africano a doença é conhecida como "nagana" ou "secadeira", termo genérico que abrange a infecção por T.vivax, T.congolense $e$ T.brucei (Gutierrez et al., 2006).

No Brasil, T.vivax foi diagnosticado no estado do Pará em 1972, infectando búfalos (Shaw \& Lainson, 1972). Em seguida, o protozoário foi detectado nos estados do Amapá (Serra-Freire, 1981), Mato Grosso (Silva et al., 1996; Osório et al., 2008), Tocantins (Linhares et al., 2006), Paraíba (Batista et al., 2008), Maranhão (Guerra et al., 2008), Minas Gerais (Carvalho et al., 2008; Frange, 2013; Meneses, 2011; Germano et al., 2017a), Rio Grande do Sul (Silva et al., 2009), São Paulo (Cadioli et al., 2012), Pernambuco (Pimentel et al., 2012) e Goiás (Barbosa et al., 2015; Bastos et al., 2017).

A doença causa grandes perdas econômica tais como: redução na produção leiteira, queda no desempenho produtivo, gastos com tratamento e honorários veterinários e mortes de animais, interferindo assim no lucro da produção. No estado de Minas Gerais, Carvalho et al. (2008) relataram uma redução de $27 \%$ na produção de leite e queda de $45 \%$ na taxa de prenhez em um surto da doença, e no estado de Goiás, $25 \%$ de queda na produção de leite foi observada quatro dias após o início de um surto (Barbosa et al., 2015).

O objetivo desta revisão é reunir e discutir informações a respeito da tripanossomose bovina, bem como os aspectos clínicos e patológicos, os fatores de riscos, as técnicas de diagnósticos, tratamento e controle da doença, gerando informações pertinentes para acadêmicos, profissionais e produtores rurais.

\section{Epidemiologia}

A epidemiologia da tripanossomose depende das interações entre parasito, vetor e hospedeiro, podendo mudar de acordo com alterações antropogênicas no ambiente, portanto, o impacto da tripanossomose bovina não é constante, mas pode mudar ao longo do tempo como resultados de mudanças de manejo ou de ambiente (Van den Bossche et al., 2010; Dagnachew \& Bezie, 2015).

No Oeste da África, T.vivax, é considerado o mais patogênico e importante hemoparasita de bovinos, principalmente em áreas ocupadas pelo seu vetor biológico, a mosca tsé-tsé (Gardiner, 1989).

A doença foi introduzida na América do Sul através da importação de gado vindo do continente Africano, provavelmente no final do século XIX. No continente americano o parasita se adaptou á transmissão mecânica por dípteros hematófagos como Stomoxy sp. e Tabanus sp. (Dabus et al., 2011).

No Brasil, T.vivax foi inicialmente diagnosticado no estado do Pará por Shaw \& Lainson (1972), desde então a situação epidemiológica, com exceção das regiões Norte e do Pantanal, onde a doença tem caráter endêmico, é dada, em sua maioria, pelo relato de surtos ou de trabalhos epidemiológicos bastante pontuais.

\section{Fatores de risco e transmissão de T.vivax}

Dentre os fatores que agravam a ocorrência da doença incluem, a presença de vetores mecânicos como tabanídeos (mutucas) e Stomoxys sp. (mosca-dos-estábulos) (Batista et al., 2007; Carvalho et al., 2008), o compartilhamento de agulhas entre bovinos, para aplicação de vacinas ou medicamentos, o que geralmente acontece pela aplicação de ocitocina em bovinos de aptidão leiteira, em que se há costume de utilizar a mesma agulha em todos os animais. Outro fator importante que favorece a ocorrência da enfermidade é o trânsito de animais de regiões onde o parasito está presente para regiões que permanecem livres ou vice-versa (Cadioli et al., 2012; Pimentel et al., 2012; Meneses, 2011).

$\mathrm{Na}$ América do Sul, a transmissão ocorre de forma mecânica por insetos hematófagos e por fômites como, por exemplo, seringas e agulhas contaminadas onde tripomastigotas são diretamente inoculadas do hospedeiro mamífero ao outro e por uma possível transmissão transplacentária (Dabus et al., 2011; Batista et al., 2012).

\section{Sinais clínicos e alterações patológicas}

Animais infectados podem apresentar-se assintomáticos e evoluir da fase aguda para a crônica (Osório et al., 2008). Bovinos infectados demonstram alta parasitemia durante a fase aguda 
apresentam sinais clínicos poucos específicos, tais como, redução na produção leiteira, apatia, anemia intensa, febre, fraqueza, perda de peso, linfonodos palpáveis aumentados, frequências cardíaca e respiratória aumentadas, mucosas apresentando petéquias e equimoses, abortos, sinais neurológicos e até morte (Batista et al., 2007; Carvalho et al., 2008; Cadioli et al., 2012; Frange, 2013; Germano et al., 2017b).

Devido a estes sinais inespecíficos, a doença pode ser confundida com outras hemoparasitoses tais como o complexo tristeza parasitária bovina causada pela Babesia sp., Anaplasma sp. ou verminoses, que causem anemia, dificultando o diagnóstico definitivo, devendo as mesmas serem consideradas no diagnóstico diferencial da tripanossomose (Wells, 1984; Paiva et al., 2000; Batista et al., 2007; Gonzatti et al., 2014).

Outra possibilidade é que ocorram animais assintomáticos, que são especialmente preocupantes, pois podem prejudicar programas de controle que dependem da identificação da doença através de sua manifestação clínica, estes animais são importantes fontes de infecção, disseminando a doença por todo o rebanho (Berthier et al., 2016). Além disto, alguns animais assintomáticos ou com doença subclínica apresentam exames parasitológicos negativos, pois em fases assintomáticas, os tripanosomas podem não estar presentes no sangue e serem encontrarem em regiões extravasculares como linfonodos (Hoare, 1972). Nesses casos, a pesquisa de anticorpos anti-T.vivax poderia auxiliar na identificação destes hospedeiros (Cuglovici et al., 2010).

Apesar da infecção não gerar lesões patognomônicas, normalmente os principais achados macroscópicos na necropsia são edema na região da barbela, palidez de carcaça, hidrotorax, líquido ascítico de coloração ictérica e linfoadenomegalia. Ademais, hepatoesplenomegalia, nefrite intersticial multifocal e presença de liquido na cavidade pericárdica (Carvalho et al., 2008; Germano et al., 2017b). No sistema nervoso central, verifica-se meningite e mielite com manguitos perivasculares, predomínio de células mononucleares e macrófagos, associados à presença de malácia e células Gitter (Batista et al., 2007).

\section{Diagnóstico}

O diagnóstico de T.vivax pode ser realizado de diversas formas, com variações na sensibilidade e especificidade dos testes, além de diferenças entre os métodos dependendo do estágio da doença. Usualmente, é feito por uma combinação entre o quadro clínico e técnicas parasitológicas, sorológicas e moleculares (Meneses, 2011).

As técnicas de diagnóstico parasitológicas são mais as utilizadas para identificar T.vivax a campo sendo de fácil execução e rapidez no reconhecimento dos trypanosomas, principalmente quando a parasitemia da amostra é alta (Frange, 2013; Gonzatti et al., 2014; Campos et al., 2015). Dentre estas, se destacam a técnica da gota espessa (Silva et al., 2002), técnica do Microhematócrito ou Woo (Woo, 1970) e por fim a técnica do Buffy Coat (Murray et al., 1977). Os testes sorológicos mais utilizados para o diagnóstico de tripanossomose destacam-se a Reação de Imunofluorescência Indireta (RIFI) e o Ensaio de Imunoabsorção Enzimático (ELISA). Já os métodos de detecção molecular o mais difundido é a Reação em Cadeia Polimerase (PCR) (Silva et al., 2002).

Métodos de diagnósticos como a RIFI podem comprovar se os animais negativos nos exames parasitológicos eram realmente negativos, pois durante fase crônica da doença, as técnicas parasitológicas demonstram baixa sensibilidade, devido à baixa parasitemia, e flutuante, sendo a técnica sorológica importante na complementação dos exames parasitológicos, tornando-se importante a associação de ambas as técnicas (Frange, 2013).

\section{Tratamento}

As drogas consideradas efetivas no tratamento de infecção por T.vivax incluem aceturato de diminazeno e cloreto de isometamídio (Gonzatti et al., 2014). Portanto, o desenvolvimento de resistência é uma ameaça para o controle de tripanossomose, sendo de grande importância realizar o diagnóstico a fim de identificar o agente envolvido, evitando o estabelecimento de resistência aos antiparasitários fazendo o uso racional das drogas tripanocidas permitindo um eficiente tratamento ou profilaxia da doença (Stevenson et al., 2000; Desquesnes, 2004; Sow et al., 2012; Frange 2013).

Entretanto, o tratamento não assegura a eliminação do parasito, há relatos que as infecções por tripanosoma possam ser reativadas por alguma situação de estresse como transporte, infecção por outros hemoprotozoários ou outras doenças (Linhares et al., 2006). Contudo, animais tratados 
continuam susceptíveis a novas infecções, pois os anticorpos para o parasito desaparecem dentro de um período de até dois anos (Gardiner \& Wilsom, 1987).

\section{Controle de tripanossomose}

Para o controle efetivo da doença, além do controle de vetores, evitar o uso compartilhado de agulhas e o controle dos animais que se movem de áreas endêmicas para áreas não endêmicas da doença. Chama-se a atenção também para uma rotina de exames a fim de identificar o agente envolvido e fazer o tratamento pontual de animais parasitados (Batista et al., 2007; Carvalho et al., 2008; Cadioli et al., 2012; Frange, 2013; Meneses, 2011; Germano et al., 2017a).

\section{Conclusão}

Conclui-se que a tripanossomose está disseminada no país, podendo causar sérios problemas em rebanhos susceptíveis. A falta de diagnóstico, o desconhecimento por parte dos produtores e profissionais é um agravo para sua ocorrência. Medidas de biosseguridade devem ser adotadas nas propriedades a fim de evitar surtos e perdas econômicas provocadas pela doença.

\section{Referências bibliográficas}

Adam, Y., Marcotty, T., Cecchi, G., Mahama, C. L., Solano, P., Bengaly, Z. \& Van den Bossche, P. 2012. Bovine trypanosomosis in the Upper West Region of Ghana: entomological, parasitological and serological cross-sectional surveys. Research in Veterinary Science, 92(3), 462-468.

Barbosa, J. C., Bastos, T. S. A., Rodrigues, R. A., Madrir, D. M. C., Faria, A. M., Bessa, L. C. \& Linhares, G. F. 2015. Primeiro surto de tripanossomose bovina detectado no estado de Goiás, Brasil. Ars Veterinaria, 31(2), 100.

Bastos, T. S. A., Faria, A. M., Madrid, D. M. C., Bessa, L. C., Linhares, G. F. C., Fidelis Junior, O. L., Lopes, W. D. Z. 2017. First outbreak and subsequent cases of Trypanosoma vivax in the state of Goiás, Brazil. Revista Brasileira de Parasitologia Veterinária, 26(3), 366-371.

Batista, J. S., Riet-Correa, F., Teixeira, M. M., Madruga, C. R \& Maia, T. F. 2007. Trypanosomiasis by Trypanosoma vivax in cattle in the Brazilian semiarid: Description of an outbreak and lesions in the nervous system. Veterinary Parasitology, 143(2), 174-181.
Batista, J. S., Bezerra, F. S. B., Lira, R. A., Carvalho, J. R. G., Rosado Neto, A. M., Petri, A. A. \& Teixeira, M. M. G. 2008. Aspectos clínicos, epidemiológicos e patológicos da infecção natural em bovinos por Trypanosoma vivax na Paraíba. Pesquisa Veterinária Brasileira, 28(1), 63-69.

Batista, J. S., Rodrigues, C. M., Olinda, R. G., Silva, T. M., Vale, R. G., Câmara, A. C., Teixeira, M. M. 2012. Highly debilitating natural Trypanosoma vivax infectious in Brazilian calves: epidemiology, pathology, and probable transplacental transmission. Parasitology Research, 110(1), 73-80.

Berthier, D., Breniére, S. F., Bras-Gonçalves, R., Lemesre, J. L., Jamonneau, V., Solano, P., ... Bucheton, B. 2016. Tolerance to trypanosomatids: a threat, or a key for disease elimination?. Trends in Parasitology, 32(2), 157-68.

Cadioli, F., De Barnabé, P.A., Machado, R., Teixeira, M. C. A., André, M. R., Sampaio, P. H., ... Marques, L. C. 2012. First report of Trypanosoma vivax outbreak in dairy cattle in São Paulo state, Brazil. Revista Brasileira de Parasitologia Veterinária, 21(2), 118-124.

Campos, M. G. S., Facury Filho, E. J., Carvalho, A. U., Ribeiro, M. F. B. \& Uribe, J. A.Z. 2015. Utilização de água de coco (Cocos nucifera) industrializada como conservante para Trypanosoma vivax. Biológico, 77(2), 1-235.

Carvalho, A. U., Abrão, D. C., Facury Filho, E. J., Paes, P. R. O. \& Ribeiro, M. F. B. 2008. Ocorrência de Trypanosoma vivax no estado de Minas Gerais. Arquivo Brasileiro de Medicina Veterinária e Zootecnia, 60(3), 769771.

Cuglovici, D. A., Bartholomeu, D. C., ReisCunha, J. L., Carvalho, A. U. \& Ribeiro, M. F. 2010. Epidemiologic aspects of an outbreak of Trypanosome vivax in a dairy cattle herd in Minas Gerais state, Brazil. Veterinary Parasitology, 169(3-4), 320-326.

Dabus, D. M. M., Campos, D. F. \& Neves, M. F. 2011 Trypanosoma vivax. Revista Eletrônica Científica da UERGS., 2161), 12.

Dagnachew, S. \& Bezie, M. 2015. Review on Trypanosoma vivax. African Journal of Basic \& Applied Sciences, 7(1), 41-64.

Desquesnes, M. 2004. Livestock trypanosomes and their vectors in Latin America. OIE World Organization for Animal Helth. Paris. 
Frange, R. C. C. Tripanossomíase em vacas na microrregião de Uberaba - MG: estudo soroepidemiológico e relato de surto. 2013.Universidade de Uberaba, Uberaba - MG, 2013.

Gardiner, P. R. 1989. Recent studies of the biology of Trypanosoma vivax. Advances in Parasitology, 28, 229-317.

Gardiner, P. \& Wilson, A. 1987. Trypanosoma (Duttonella) vivax. Parasitology Today, 3, 4952, 1987.

Germano, P. H. V., Edler, G. E. C., Silva, A. A. \& Lopes, L. O. 2017a. Prevalência de Tripanosoma vivax em bovinos no município de Patos de Minas/MG. Revista Acadêmica: Ciência Animal, 15(2), 433-434.

Germano, P. H. V., Silva, A. A., Edler, G. E. C. \& Lopes, L. O. 2017b. Aspectos patológicos e clínicos de uma bezerra Holandesa infectada naturalmente por Tripanosoma sp. na região do Alto Paranaíba/MG. Revista Acadêmica: Ciência Animal, 15(2), 609-610.

Gutierrez, C., Corbera, J. A., Morales, M. \& Buscher, P. Trypanosomosis in goats: current status. Annals of the New York academy of sciences, v. 1081.P. 300-310, 2006.

Guerra, R. M. S. N. C., Feitosa Júnior, A. B., Santos, H. P., Abreu-Silva, A. L. \& Santos, A. C. G. 2008. Biometry of Trypanosoma vivax found in a calf in the state of Maranhão, Brazil. Ciência Rural, 38, 833-835.

Gonzatti, M. I., González-Baradat, B., Aso, P. M. \& Reyna-Bello. 2014. Trypanosoma (Duttonella) vivax and Trypanosomosis in Latina America: Secadera/Huequera/Cacho Hueco. In: Magez, S. \& Radwanska, M. Trypanosomes and Trypanosomiasis. Springer-Verlag Wien, London, United Kingdom.

Hoare, C. A. 1972. The Trypanosomes of Mammals. Blackwell Scientific Publications, Oxford, USA.

Linhares, G. F. C., Dias Filho, A. M. R., Fernandes, P. R. \& Duarte, S. C. 2006. Tripanossomíase em bovinos no município de Formoso do Araguaia, Tocantins (relato de caso). Ciência Animal Brasileira, 7(4), 455-460.

Meneses, R. M. Tripanossomose bovina em Minas Gerais, 2011: soroprevalência e fatores de risco. Universidade Federal de Minas Gerais, Belo Horizonte- MG.
Murray, M., Murray, P. K. \& Mcintyre, W. I. M. 1977. An improved parasitological technique for the diagnosis of African trypanosomisis.Trans. Royal Society of Tropical Medicine and Hygiene, 71, 317-318.

Osório, A. L. A. R., Madruga, C. R., Desquesnes, M., Soares, C. O., Ribeiro, L. R. R. \& Costa, S. C. G. 2008. Trypanosoma (Duttonela) vivax: its biology, epidemiology, pathogenesis, and introdution in the New World - A Review. Memórias do Instituto Oswaldo Cruz, 103, 113.

Paiva, F., Lemos, R. A. A., Nakasato, L., Mori, A. E., Brum, K. B. \& Bernardo, K. C. 2000. Trypanosoma vivax em bovinos do Estado de Mato Grosso do Sul, Brasil. I. Acompanhamento clínico, laboratorial e anatomopatológico de rebanhos infectados. Revista Brasileira de Parasitologia Veterinária, 9, 135-141.

Pimentel, D. S., Ramos, C. A. N., Ramos, R. A. N., Araújo, F. R., Borba, M. L., Faustino, M. A. G. \& Alves, L. C. 2012. First report and molecular characterization of Trypanosoma vivax in cattle from state of Pernambuco, Brazil. Veterinary Parasitology, 185(2-4), 286-289.

Serra-Freire, N. M. 1981. Oiapoque-outro foco de Trypanosoma vivax no Brasil. Revista Brasileira de Medicina Veterinária, 4, 30-31.

Silva, R. A. M. S., Da Silva, J.A., Schneider, R.C., Freitas, J., Mesquita, D., Mesquita, T., Pereira, M. E. B. 1996. Outbreak of trypanosomiasis due to Trypanosoma vivax (Ziemann, 1905) in bovines of the Pantanal, Brazil. Memórias do Instituto Oswaldo Cruz, 91, 561-562.

Silva, R. A. M. S., Rivera Dávila, A. M., Seidl, A. \& Ramirez, L. 2002. Trypanosoma evansi e Trypanosoma vivax-Biologia, Diagnóstico e Controle (140 p.). EMBRAPA, Corumbá, BR.

Silva, A. S., Costa, M. M., Polenz, M. F., Polenz, C. H., Teixeira, M. M. G.., Lopes, S. T. A. \& Monteiro, S. G. 2009. Primeiro registro de Trypanosoma vivax em bovinos no Estado do Rio Grande do Sul, Brasil. Ciência Rural, 39, 2550-2554.

Sow, A., Sibidé, I., Bengaly, Z., Marcotty, T., Séré, M., Diallo, A., ... Delespaux, V. 2012. Field detection of resistence to isometamidium chloride and diminazene aceturate in Trypanosoma vivax from the region of the 
Boucle du Mouhoun in Burkina Faso. Veterinary Parasitology, 187, 105-111.

Shaw, J. J. \& Lainson, R. 1972. Trypanosoma vivax in Brazil. Annals of Tropical Medicine and Parasitology, 66, 25-32.

Stevenson, P., Okeck, G., Mwendia, C. \& Sones, K. R. 2000. Comparison of the isometamidium-based trypanocidal drugs Samorin ${ }^{\circledR}$ and Veridium ${ }^{\circledR}$ in cattle under field conditions at Nguruman, Kenya. Acta Tropica, 77, 195-201.

Van Den Bossche, P., De La Rocque, S., Hendrickx, G. \& Bouyer, J. A. 2010. Changing environment and the epidemiology of tsetsetransmitted livestock trypanosomiasis. Trends in Parasitology, 26(5), 236-43.
Wells, E. 1984. Animal trypanosomiasis in south America. Preventive Veterinary Medicine, 2, 31-41.

Woo, P. T. K. 1970. The haematocrit centrifuge technique for the diagnosis of African trypanosomosis. Acta Tropica, 27, 384-386.

Recebido: 21 Mai., 2018.

Aprovado: 18 Jun., 2018

Publicado: 18 Jul., 2018

Licenciamento: Este artigo é publicado na modalidade Acesso Aberto sob a licença Creative Commons Atribuição 4.0 (CC-BY 4.0), a qual permite uso irrestrito, distribuição, reprodução em qualquer meio, desde que o autor e a fonte sejam devidamente creditados. 